\title{
Prevalence and risk factors for post-traumatic stress, anxiety, and depression in sepsis survivors after ICU discharge
}

\author{
Allan J. Calsavara, ${ }^{1,2}$ iD Priscila A. Costa, ${ }^{3}$ Vandack Nobre, ${ }^{2}$ Antonio L. Teixeira ${ }^{2,4}$ \\ ${ }^{1}$ Escola de Medicina, Universidade Federal de Ouro Preto (UFOP), Ouro Preto, MG, Brazil. ${ }^{2}$ Programa de Pós-Graduação em Ciências da \\ Saúde, Infectologia e Medicina Tropical (PPG-IMT), Universidade Federal de Minas Gerais (UFMG), Belo Horizonte, MG, Brazil. ${ }^{3}$ Faculdade \\ de Medicina, UFMG, Belo Horizonte, MG, Brazil. ${ }^{4}$ Department of Psychiatry and Behavioral Sciences, McGovern Medical School, University of \\ Texas Health Science Center at Houston, Houston, TX, USA.
}

\begin{abstract}
Objective: Sepsis survivors present a wide range of sequelae; few studies have evaluated psychiatric disorders after sepsis. The objective of this study was to define the prevalence of and risk factors for anxiety, depression and post-traumatic stress disorder (PTSD) symptoms in sepsis survivors.

Method: Anxiety, depression and post-traumatic stress symptoms in severe sepsis and septic shock survivors $24 \mathrm{~h}$ and 1 year after intensive care unit (ICU) discharge were assessed using the Beck Anxiety/Depression Inventories and the PTSD Checklist-Civilian Version. Differences in psychiatric symptoms over time and the influence of variables on these symptoms were calculated with marginal models.

Results: A total of 33 patients were enrolled in the study. The frequencies of anxiety, depression and PTSD $24 \mathrm{~h}$ after ICU discharge were $67 \%$, 49\%, and 46\%, respectively and, among patients reevaluated 1 year after ICU discharge, the frequencies were $38 \%, 50 \%$, and $31 \%$, respectively. Factors associated with PTSD included serum S100B level, age, and Informant Questionnaire on Cognitive Decline in the Elderly (IQCODE) score. Factors associated with depression included patient age and cumulative dose of dobutamine. IQCODE score and cumulative dose of haloperidol in the ICU were associated with anxiety after ICU discharge.

Conclusion: Patients who survive sepsis have high levels of psychiatric symptoms. Sepsis and associated treatment-related exposures may have a role in increasing the risk of subsequent depression, anxiety, and PTSD.
\end{abstract}

Keywords: Sepsis; anxiety; depression; post-traumatic stress disorder; intensive care units

\section{Introduction}

Sepsis is defined as a life-threatening organ dysfunction caused by a dysregulated host response to infection. ${ }^{1}$ Worldwide, more than 19 million patients have severe sepsis and 5 million people die from sepsis each year, with the majority occurring in low- and middle-income countries. $^{2}$ Among survivors, a high percentage experience long-term physical, cognitive and behavioral sequelae. ${ }^{3}$

Sepsis survivors present a wide range of sequelae. Six months after discharge, one-third of sepsis survivors do not return to independent living, which indicates the disruption caused by a septic event. ${ }^{4}$ Most studies have focused on long-term organic (i.e., lung, heart, etc.) sequelae, while few studies have evaluated psychiatric disorders after sepsis. Among psychiatric disorders, anxiety, depression, and post-traumatic stress disorder (PTSD) can develop in sepsis survivors and have a profound effect on their everyday functioning and chance

Correspondence: Allan J. Calsavara, Escola de Medicina, Universidade Federal de Ouro Preto, Rua Dois, Campus Morro do Cruzeiro, CEP 35400-000, Ouro Preto, MG, Brazil.

E-mail: allancalsavara@ ufop.edu.br

Submitted Apr 09 2020, accepted Jun 19 2020, Epub Oct 072020. of returning to work. ${ }^{5}$ A study of severe sepsis survivors and their relatives showed that two-thirds reported clinically relevant PTSD and about one-third of the patients showed abnormal levels of anxiety and depression. ${ }^{6}$

Our aim was to identify the prevalence and risk factors associated with the development of anxiety, depression and post-traumatic stress symptoms after severe sepsis and septic shock.

\section{Methods}

\section{Study participants}

The current investigation took place at the University Hospital of the Universidade Federal de Minas Gerais. We prospectively evaluated 658 consecutive patients admitted to the intensive care unit (ICU) during the period of investigation. Based on the diagnostic criteria of the 2001 SCCM/ESICM/ACCP/ATS/SIS International Sepsis

How to cite this article: Calsavara AJ, Costa PA, Nobre V, Teixeira AL. Prevalence and risk factors for post-traumatic stress, anxiety, and depression in sepsis survivors after ICU discharge. Braz J Psychiatry. 2021;43:269-276. http://dx.doi.org/10.1590/1516-44462020-0986 
Definitions Conference, ${ }^{7}$ the inclusion criteria were a history of severe sepsis (sepsis and concomitant acute organ dysfunction in at least one organ) or septic shock (persistent arterial hypotension unexplained by other causes, despite adequate volume resuscitation) in patients discharged from the ICU. Key exclusion criteria were: a) age under 18 years; b) pregnancy; c) less than three months of life expectancy; d) immunosuppression; e) neurological disease at the time of inclusion (epilepsy, brain tumor, neuroinfection, stroke, trauma); f) acute decompensated renal failure or g) hepatic insufficiency; and $h$ ) tracheostomy or any other condition leading to speech limitations.

An in-person interview was administered to enrolled patients $24 \mathrm{~h}$ after ICU discharge, and they were reassessed 1 year later.

\section{Demographic and clinical data}

Upon arrival at the ICU and after discharge, the following data were obtained: age, sex, race, marital status, education level, Acute Physiology and Chronic Health Evaluation II (APACHE II) score, sepsis-related organ failure assessment (SOFA) score, time until antimicrobial administration after recognition of septic shock or severe sepsis, ICU and hospital length of stay, use of mechanical ventilation, septic shock or severe sepsis diagnosis, surgery or general ICU admission, use of corticosteroids in the first $72 \mathrm{~h}$ after ICU admission, and comorbid diseases. The cumulative dose of midazolam, fentanyl, dobutamine, noradrenaline and haloperidol were monitored during the ICU stay. The treatment of all patients was carried out at the discretion of the ICU physicians.

Patient comorbidities were assessed using the Charlson comorbidity index, a scoring system that involves weighting factors based on the number and severity of ongoing diseases. This index was originally developed as a prognostic tool for patients admitted to a general medical service with different medical conditions.

Due to the impossibility of obtaining a baseline cognitive performance score for patients with sepsis, premorbid cognitive impairment was estimated based on the Informant Questionnaire on Cognitive Decline in the Elderly (IQCODE). ${ }^{8}$ The IQCODE, the most widely used informant instrument, was developed to estimate a patient's cognitive decline from a pre-morbid level based on an informant's report. The IQCODE has high reliability and measures a single general factor of cognitive decline..$^{9}$ The questionnaire was applied to the informants while the patient was hospitalized.

\section{Laboratory}

Four biomarkers were measured for their potential role as biomarkers of sepsis severity or central nervous system involvement/damage: high mobility group box 1 (HMGB1), S100 calcium-binding protein B (S100B), neuron specific enolase (NSE), and brain-derived neurotrophic factor (BDNF). These biomarkers were measured at ICU discharge and around one year after discharge, with the samples run in duplicate using commercial ELISA kits according to manufacturer instructions (R\&D Systems, USA; BD Biosciences, East Rutherford, NJ, USA and BioVendor, Modrice, Czech Republic). The assessment was performed blind to the clinical status of the subjects.

\section{PTSD Checklist-Civilian Version}

The severity of PTSD symptoms at ICU discharge and 1 year later was assessed with the PTSD Checklist-Civilian Version (PCL-C). ${ }^{10}$ This instrument includes questions about symptoms from the following PTSD clusters: five from the intrusive cluster, seven from the avoidant cluster, and five from the arousal cluster. Symptom severity is rated on a five-point Likert scale. Clinically significant PTSD symptoms can be ascertained from test scores through an algorithm that considers a score of 3 or more on at least one intrusive symptom, three avoidant symptoms and two arousal symptoms as consistent with the DSM criteria.

\section{Beck Depression Inventory-II}

The Beck Depression Inventory-II is one of the most commonly used self-report measures to assess depressive symptoms ${ }^{11}$ It consists of 21 items rated on a 4-point scale (0 to 3 ) that indicates different levels of symptom severity experienced over the past week. Total scores range from 0 to 63 . The scale measures core and associated symptoms of depression severity, with cutoff scores that distinguish minimal (0 to 13), mild (14 to 19), moderate (20 to 28), and severe (29 and greater) depression. In this study, the cutoff point was set at 21 .

\section{Beck Anxiety Inventory}

The Beck Anxiety Inventory is a 21-item self-report measure designed to assess the presence and severity of anxiety symptoms in the past week and distinguish these symptoms from depressive symptoms. ${ }^{12}$ Specifically, the questionnaire refers to both cognitive and somatic symptoms of anxiety. Each item is scored on a 4-point scale, from 0 (not at all) to 3 (severe, barely tolerable), and total scores range from 0 to 63 , with higher scores corresponding to higher levels of anxiety. In this study, scores over 15 points were considered indicative of anxious symptoms.

\section{Statistical analysis}

The participants' demographic and clinical characteristics were summarized as counts and percentages for dichotomous items, and means and SD for quantitative items. The Shapiro-Wilk test was used to assess data normality. Student's $t$-test, the Mann-Whitney $U$ test, and the chisquare test were used when appropriate. Differences in neuropsychiatric symptoms over time (controlling for possible confounding factors) and the influence of the variables on neuropsychiatric symptoms were calculated using marginal models. Stepwise regression was used to identify a useful subset of predictors of psychiatric syndromes. First a univariate marginal linear model was 
performed using the forward stepwise method to select variables that correlated with anxiety, depression or PTSD symptoms in patients after sepsis. Variables with $p<0.15$ were tested for independence in multiple marginal regression analysis using the backward stepwise method. A p-value $<0.05$ was considered statistically significant. Quasi-likelihood under independence model criterion values were used to estimate a model's likelihood of predicting future values. The analyses were carried out in R version 3.2.2 $2^{13}$ and SPSS version 19.0.

\section{Ethics statement}

The study protocol was approved by the local ethics committee (0319.0.203.000 - 11), and written informed consent was obtained from all participants or their representative prior to testing. In addition, all procedures were performed in accordance with the relevant guidelines and regulations.

\section{Results}

Of the 658 consecutive patients admitted to the ICU, 80 had severe sepsis or septic shock during their ICU stay. A total of 33 patients were considered eligible for the study and were evaluated $24 \mathrm{~h}$ after ICU discharge. Sixteen of these patients $(48 \%)$ were re-evaluated approximately one year after discharge (Figure 1).

Table 1 shows the baseline characteristics of septic patients enrolled in this study. Re-interviews were completed at a mean of 393 days after ICU discharge. The patients' mean age at hospitalization was 49.0 years (standard deviation $[S D]=15.2$ ), and they had a mean of IQCODE score of 3.1 (SD $=0.5$ ). Invasive mechanical ventilation was used in $42 \%$ of the patients during their ICU stay.

A comparison of the demographic, clinical and laboratory characteristics between patients re-evaluated and not re-evaluated after one-year follow-up showed no significant between-group differences in baseline characteristics (Table S1, available as online-only supplementary material).

Figure $2 \mathrm{~A}$ shows the frequencies of clinically significant post-ICU PTSD, depressive, and anxiety symptoms $24 \mathrm{~h}$ and 1 year after ICU discharge. At $24 \mathrm{~h}$ after ICU discharge (mean ICU length of stay: 9.4 days, SD $=7.9$ ), $46 \%(95 \%$ confidence interval $[95 \% \mathrm{Cl}] 29-63)$ of the patients had significant PTSD symptoms, 49\% $(95 \% \mathrm{Cl}$ 31-66) had significant depressive symptoms, and $67 \%$ $(95 \% \mathrm{Cl} 51-83)$ had moderate to severe anxiety. One year after ICU discharge (mean $=379.2$ days, $S D=149.0)$, the frequency of significant PTSD and anxiety symptoms had decreased to $31 \%(95 \% \mathrm{Cl} 9-54)$ and $38 \%(95 \% \mathrm{Cl} 14-62)$, respectively, while the prevalence of significant depressive symptoms was $50 \%(95 \% \mathrm{Cl} 26-75)$.

Most patients had significant PTSD, depression, or anxiety symptoms $24 \mathrm{~h}$ after ICU discharge. Only $24.2 \%$ had no meaningful psychiatric symptoms. By one year after ICU discharge, the frequency of significant PTSD and anxiety symptoms decreased and the prevalence of patients with no psychiatric symptoms increased. On the other hand, the prevalence of depression increased slightly in this period (Figure $2 \mathrm{~B}$ and $2 \mathrm{C}$ ).

The following courses of significant PTSD could be identified in the study: $68.8 \%$ of patients were resilient, i.e., did not develop symptoms (31.3\%) or recovered (37.5\%); $31.2 \%$ had delayed onset, i.e., developed symptoms after hospital discharge $(12.5 \%)$, or had a persistent course (18.7\%). Among patients with significant depression, half were resilient $(25 \%)$ or recovered $(25 \%)$; the other half had delayed-onset $(12.5 \%)$ or a persistent course $(37.5 \%)$. Of the patients with significant anxiety, $62.5 \%$ were resilient $(25 \%)$ or recovered $(37.5 \%), 37.5 \%$ showed a delayedonset $(12.5 \%)$ or persistent course (25\%). Only $18.75 \%$ of the patients had no psychiatric disorder during the study (Figure 2B and 2C).

In univariate analysis, PTSD was associated with the following baseline characteristics: age $(p=0.014)$, nonwhite race $(p=0.048)$, Charlson comorbidity index ( $p$ $=0.097)$, IQCODE $(p=0.004)$, as well as time of the first antibiotic dose $(p=0.035)$, serum S100B levels $(p=0.046)$, and NSE $(p=0.070)$. PTSD was not significantly associated $(p>0.15)$ with sex, education level, marital status, hospital or ICU length of stay (LOS), severe sepsis vs. septic shock diagnosis at ICU admission, APACHE II and SOFA score, mechanical ventilation status, cumulative dose of midazolam, fentanyl, noradrenaline, dobutamine or haloperidol during ICU stay, corticosteroid use in the first $72 \mathrm{~h}$, or serum level of HMGB1 and BDNF (Table S2, available as online-only supplementary material). After multivariate analysis, the presence of PTSD was associated with S100B level, age and IQCODE score (Table 2).

Table 3 (and Table S3, available as online-only supplementary material) shows the results of the stepwise regression analysis of factors associated with depressive symptoms. In univariate analysis, significant depressive symptoms were associated with age $(p=0.021)$, Charlson comorbidity index $(p=0.089)$, APACHE II score $(p=0.009)$, and cumulative dose of dobutamine $(p=$ 0.082 ) (Table S3). After adjusted analyses, the presence of significant depressive symptoms was associated with age and dobutamine (Table 3).

We also evaluated the association between anxiety and independent variables related to baseline, clinical, laboratory, and intra-ICU characteristics of patients with severe sepsis or septic shock (Table 4 and Table S4, available as online-only supplementary material). Unadjusted analyses showed an association between IQCODE score $(p=0.004)$, hospital length of stay $(p=0.111)$, cumulative dose of fentanyl $(p=0.006)$, haloperidol $(p=0.023)$ and noradrenaline $(p=0.118)$, corticosteroid use in the first 72 $h(p=0.134)$, and serum levels of NSE $(p=0.117)$ and BDNF $(p=0.067)$ (Table S4). In the multivariate analysis, the presence of significant anxiety symptoms was associated with IQCODE and cumulative haloperidol dose (Table 4).

\section{Discussion}

In this study, we found that depression, anxiety and PTSD symptoms are common in severe sepsis and septic-shock 


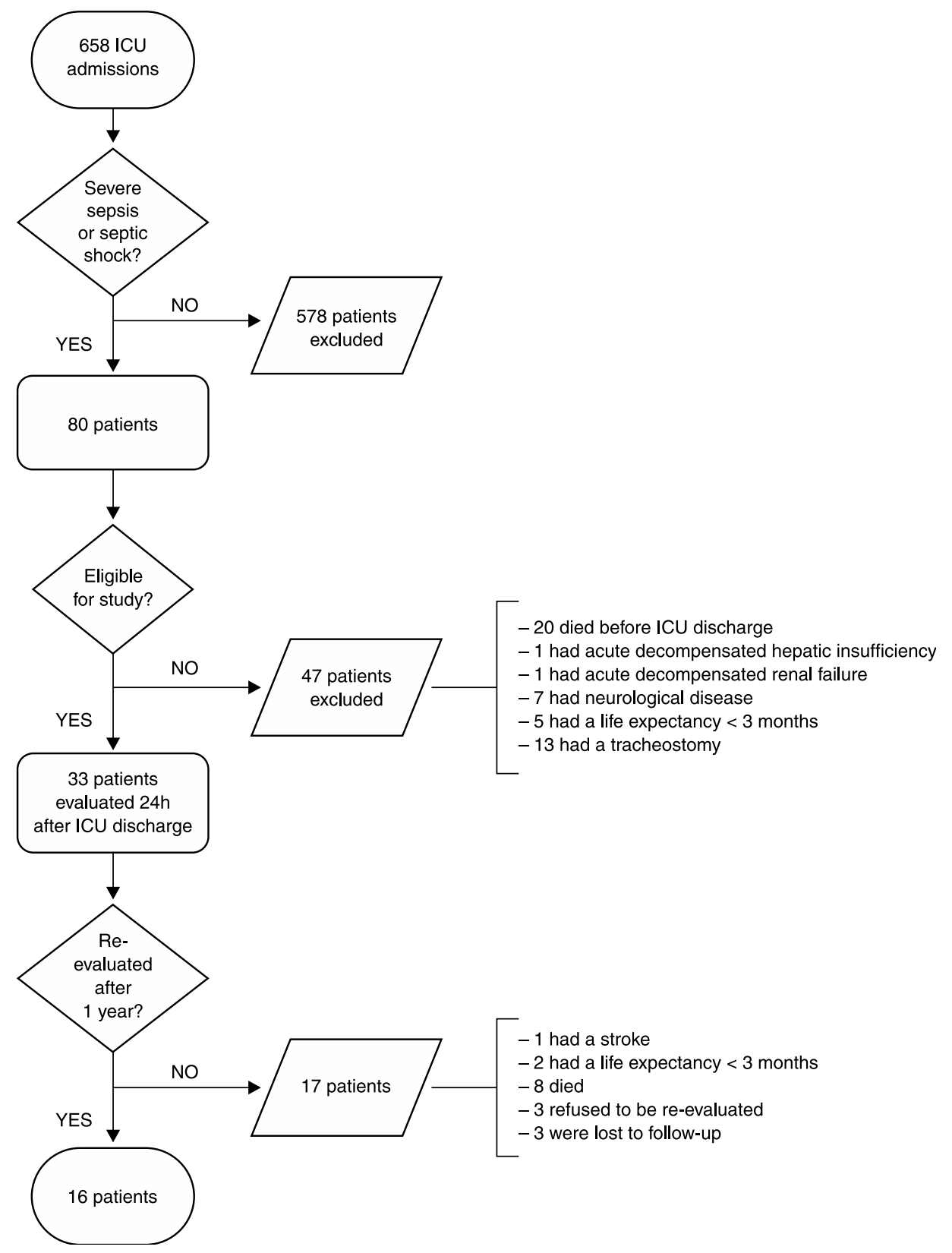

Figure 1 Flowchart of patient selection. ICU = intensive care unit.

ICU survivors, and these symptoms tend to persist even after one year. Interestingly, high serum S100B levels were associated with a higher risk of PTSD symptoms, while age and IQCODE scores were protective factors. Cumulative dobutamine dose during the ICU stay was associated with a higher risk of depression, while age was a protective factor. Finally, higher IQCODE scores and cumulative haloperidol dose were associated with decreased anxiety.

More than three-quarters of the patients in this study had clinically meaningful psychiatric symptoms during follow-up. Among the evaluated psychiatric symptoms, depression was the most prevalent, affecting $50 \%$ of the patients one year after discharge. This result was consistent with previous studies of post-ICU septic patients, in which the depression prevalence ranged between $10-58 \% .{ }^{14-16}$ Among the risk factors for depressive symptoms, we found that each 10-year increase in patient age lowered the risk of depressive symptoms by $56 \%$. It is unclear why older age had a protective effect against depression in this context. Considering that most patients were not older adults (a period when depression is associated with negative outcomes, such as cognitive impairment), a possible explanation is that older adults are more likely to cope with adverse situations (e.g., hospitalizations) using acceptance and adaptive strategies, therefore reducing the overall emotional burden and related effects. ${ }^{17}$ We also found a positive association between cumulative dobutamine dose and depressive symptoms. Dobutamine is a $\beta 1$-adrenoceptor ( $\beta 1 \mathrm{AR})$ agonist. $\beta 1 \mathrm{AR}$ 
Table 1 Baseline and clinical characteristics of septic patients $(n=33)$

\begin{tabular}{|c|c|}
\hline \multicolumn{2}{|l|}{ Patient baseline characteristics } \\
\hline Age, mean (SD) & $49.0(15.2)$ \\
\hline Sex (female) & $19(57.6)$ \\
\hline Nonwhite & $20(60.6)$ \\
\hline$>$ High school graduate & $6(18.2)$ \\
\hline Married/partnered & $12(36.4)$ \\
\hline Charlson comorbidity index, mean (SD) & $2.4(2.3)$ \\
\hline IQCODE, mean (SD) & $3.1(0.5)$ \\
\hline \multicolumn{2}{|l|}{ Clinical characteristics upon admission } \\
\hline Hospital LOS (days), mean (SD) & $49.9(50.3)$ \\
\hline ICU LOS (days), mean (SD) & $9.4(7.9)$ \\
\hline \multicolumn{2}{|l|}{ Admission diagnosis } \\
\hline Cardiovascular & $2(6.1)$ \\
\hline Pulmonary & 7 (21.2) \\
\hline Infectious disease & $19(57.6)$ \\
\hline Other & $4(12.1)$ \\
\hline \multicolumn{2}{|l|}{ ICU characteristics } \\
\hline \multicolumn{2}{|l|}{ Mechanically ventilated } \\
\hline First $72 \mathrm{~h}$ & $20(60.6)$ \\
\hline During ICU stay & $21(63.6)$ \\
\hline APACHE II, mean (SD) & $16.5(7.2)$ \\
\hline SOFA, mean (SD) & $7.0(3.2)$ \\
\hline Surgical admission & $4(12.1)$ \\
\hline $\begin{array}{l}\text { Time to antibiotic administration ( } \mathrm{min} \text { ), } \\
\text { mean (SD) }\end{array}$ & $27.0(58.4)$ \\
\hline Septic shock & $25(75.8)$ \\
\hline \multicolumn{2}{|l|}{ Cumulative dosage of, mean (SD) } \\
\hline Midazolam (mg) & $299.8(775.4)$ \\
\hline Fentanyl $(\mu \mathrm{g})$ & $4,267.7(9,651.9)$ \\
\hline Haloperidol (mg) & $7.8(27.3)$ \\
\hline Dobutamine (mg) & $356.2(928.5)$ \\
\hline Noradrenaline $(\mathrm{mg})$ & $49.1(94.8)$ \\
\hline Corticosteroids in the first $72 \mathrm{~h}$ & $6(18.2)$ \\
\hline
\end{tabular}

Results presented as $\mathrm{n}(\%)$, unless otherwise specified. APACHE II = Acute Physiology and Chronic Health Evaluation II; ICU = intensive care unit; LOS = length of stay; IQCODE = Informant Questionnaire on Cognitive Decline in the Elderly; SOFA = sepsis-related organ failure assessment.

is the most abundant subtype in the mammalian brain, and many studies have reported alterations in density and/or sensitivity of $\beta A R s$, as well as dysfunction in $\beta A R s$ signal transduction pathways, in mood disorders. ${ }^{18}$ For instance, $\beta 1 A R$ density was found to be increased in antidepressant-free subjects with depression compared to non-depressed controls, and these receptors could be down-regulated by antidepressant treatment. ${ }^{19}$ It is also known that sympathetic nervous system-driven changes are a well-known component of psychosocial stress and are at the core of the classic "fight or flight" reaction to a perceived or actual stressor. Although no previous study has specifically evaluated the sympathomimetic effect of dobutamine in mood disorders, we hypothesized that dobutamine could induce changes in the density, sensitivity and/or signal transduction of $\beta A R s$, mechanisms already implicated in mood disorders.

Previous studies using lipopolysaccharide-induced inflammatory response in healthy volunteers found that state anxiety and circulating inflammatory cytokines increase after administering lipopolysaccharide, which suggests that inflammatory mechanisms play a role in the pathophysiology of anxiety. ${ }^{20}$ No previous study has evaluated the risk factors during an ICU stay for developing post-ICU anxiety in sepsis survivors. Our data showed that anxiety was associated with IQCODE scores and cumulative haloperidol dose during ICU stay. IQ CODE scores are generally used to screen dementia in older patients, and in this study we used them as a predictor of previous cognitive decline. Lower cognitive performance according to the IQCODE was associated with lower risk of post-sepsis anxiety. Cumulative haloperidol dose during ICU stay was inversely associated with anxiety after ICU discharge. Interestingly, midazolam, a short-acting benzodiazepine, was not associated with anxiety in our patients. A previous study compared haloperidol and diazepam in anxiety treatment and found that haloperidol was more effective. ${ }^{21}$ Although antipsychotic drugs are often prescribed to patients with anxiety disorders, evidence regarding their benefits is limited. ${ }^{22,23}$

Post-ICU patients with severe sepsis or septic shock have a high prevalence of PTSD symptoms, and these symptoms appear to persist over time. Even after one year, more than one-third of the patients had PTSD symptoms. PTSD has been identified in approximately 19 to $22 \%$ of ICU survivors, and almost one quarter of patients with chronic critical illness had PTSD up to 6 months after ICU discharge. ${ }^{24,25}$ The discrepancies between these findings could be explained by the clinical characteristics of the patients (e.g., illness severity and length of ICU stay) and the re-evaluation period after ICU discharge. We believe our results are consistent with those of cohorts with more severe patients who were followed-up for a longer period.

In agreement with our findings, Sojka et al. found an association between PTSD one year after a traumatic event and serum S100B levels. ${ }^{26}$ Although $5100 B$ is mainly regarded as a brain injury biomarker, these findings highlight the complexity of the brain's reaction to stress, whether psychological or biological. ${ }^{26}$ It could be that S100B levels increased after a sepsis-related inflammatory insult to the brain, affecting its functioning and structure. A heightened pro-inflammatory state, for instance, has been demonstrated to significantly suppress hippocampal neurogenesis, leading to decreased hippocampal volume, a neuroanatomical trait that marks vulnerability to PTSD. ${ }^{27,28}$

Age also seemed to play a role in the development of PTSD symptoms, with younger patients more likely to develop them. This finding is in line with our previous assumption that younger patients would be more susceptible to social and occupational challenges after sepsis, while older patients would be more likely to adjust to limitations resulting from serious illness. ${ }^{29,30}$

PTSD is characterized by several cognitive impairments that affect memory (especially episodic memory), executive functioning, and self-referential processing. ${ }^{31-33}$ Hermelink et al. found an association between pretreatment cognitive impairment, as assessed by a neuropsychological battery, and PTSD after local or systemic treatment in cancer patients. ${ }^{34}$ Based on the IQCODE, an indirect measure of cognitive performance, our study showed that previous cognitive impairment may exert a protective effect against developing PTSD. More research 


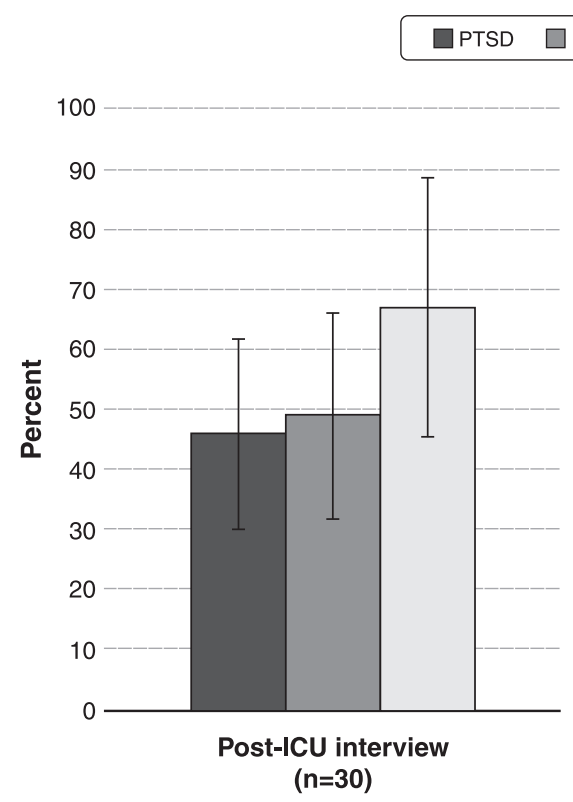

A

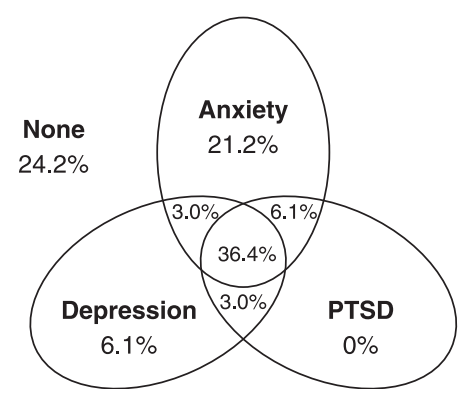

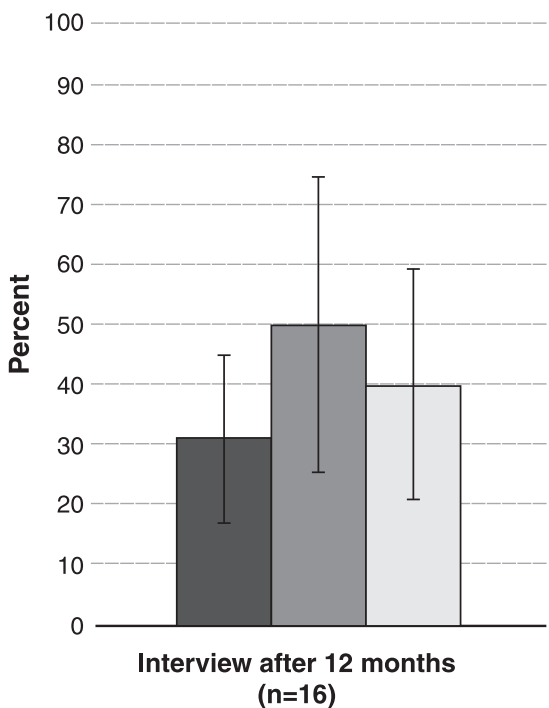

B

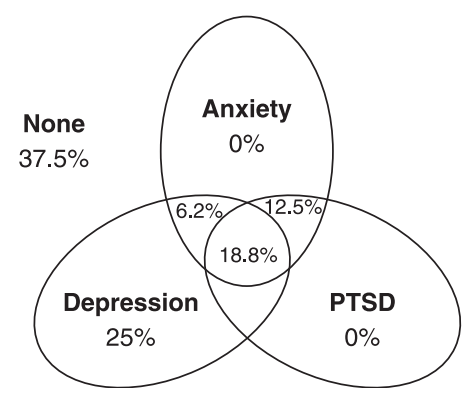

Figure 2 A) Prevalence of clinically significant PTSD, ${ }^{a}$ depressive, $^{\mathrm{b}}$ and anxiety ${ }^{\mathrm{c}}$ symptoms $24 \mathrm{~h}$ and 1 year after ICU discharge. ${ }^{\text {a }}$ Significant PTSD symptoms were defined as meeting PTSD Checklist-Civilian Version algorithm-based criteria for a DSM diagnosis of PTSD. ${ }^{b}$ Significant depressive symptoms were defined as a Beck Depression Inventory score $\geqslant 21 .{ }^{c}$ Significant anxiety symptoms were defined as a Beck Anxiety Inventory score $\geqslant 16$. Below: Venn diagram showing the prevalence of patients with significant PTSD, anxiety and depression symptoms $24 \mathrm{~h}$ (B) and 1-year (C) after ICU discharge. $\mathrm{ICU}=$ intensive care unit; PTSD = post-traumatic stress disorder.

Table 2 Adjusted associations between clinical and laboratory characteristics and post-traumatic stress disorder symptoms one year after intensive care unit admission due to severe sepsis or septic shock

\begin{tabular}{lccr}
\hline Predictor & $\beta$ & SE $\beta$ & OR $(95 \% \mathrm{Cl})$ \\
\hline S100B $(\mathrm{pg} / \mathrm{mL})$ & $0.109^{*}$ & 0.049 & $1.12(1.01-1.23)$ \\
Age/10 & $-0.573^{*}$ & 0.270 & $0.56(0.33-0.96)$ \\
IQCODE & $-1.509^{\dagger}$ & 0.535 & $0.22(0.08-0.63)$ \\
Intercept & 7.522 & 2.260 & -
\end{tabular}

$95 \% \mathrm{Cl}=95 \%$ confidence interval; IQCODE = Informant Questionnaire on Cognitive Decline in the Elderly; OR = odds ratio; SE = standard error.

Quasi-likelihood under independence model criterion $=58.32 ;{ }^{*} p<0.05,{ }^{\dagger} p<0.01$.

is needed to determine which types of cognitive impairment precede the emergence of PTSD, and conversely, to what extent cognitive control and the ability to regulate emotion before exposure to trauma results in resilience. ${ }^{33}$

Patients who survive sepsis have very high levels of psychiatric symptoms, and several authors have reported that inflammation and psychiatric disorders are associated in a synergistic bidirectional loop. ${ }^{35,36}$ Major depression is the quintessential example of this reciprocal interaction. Major depression is a risk factor for infection, possibly due to a chronic low-grade inflammatory state that leads to less efficient pathogenic defense, while infection is a risk factor for depression, which suggests that peripheral inflammation can modify a patient's mood. ${ }^{37,38}$

The mechanisms underlying the current observations are still unclear, but sepsis-related cerebrovascular injury and neuroinflammation seem to be major players in the behavioral and cognitive sequelae of sepsis. Only a few 
Table 3 Adjusted associations between clinical and laboratory characteristics and significant depressive symptoms one year after intensive care unit admission due to severe sepsis or septic shock

\begin{tabular}{lccc}
\hline Predictor & $\beta$ & SE $\beta$ & OR (95\%Cl) \\
\hline Age/10 & $-0.827^{*}$ & 0.301 & $0.44(0.24-0.79)$ \\
Dobutamine/1,000 (mg) & $0.908^{\dagger}$ & 0.423 & $2.48(1.08-5.68)$ \\
Intercept & 3.870 & 1.531 & - \\
\hline
\end{tabular}

$95 \% \mathrm{Cl}=95 \%$ confidence interval; OR = odds ratio; SE = standard error.

Quasi-likelihood under independence model criterion $=59.54,{ }^{*} p<0.05,{ }^{\dagger} p<0.01$.

Table 4 Adjusted associations between clinical and laboratory characteristics and anxiety symptoms one year after intensive care unit admission due to severe sepsis or septic shock

\begin{tabular}{lccc}
\hline Predictor & $\beta$ & SE $\beta$ & OR (95\%Cl) \\
\hline IQCODE & $-1.641^{\star}$ & 0.507 & $0.28(0.10-0.75)$ \\
Haloperidol/10 (mg) & $-2.157^{\dagger}$ & 0.749 & $0.12(0.03-0.50)$ \\
Intercept & 4.153 & 1.641 & - \\
\hline
\end{tabular}

$95 \% \mathrm{Cl}=95 \%$ confidence interval; IQCODE = Informant Questionnaire on Cognitive Decline in the Elderly; OR = odds ratio; SE = standard error.

Quasi-likelihood under independence model criterion $=59.75,{ }^{*} p<0.05,{ }^{\dagger} p<0.01$.

studies have specifically evaluated psychiatric issues after sepsis, in contrast with the significant number of studies that have examined these issues as "post-intensive care unit syndrome." It is worth mentioning that many intensive care unit patients also have sepsis and, thus, are often considered to have "post-intensive care unit syndrome." 39 Brain lesions due to inflammatory, ischemic/ hypoxic and dysglycemic insults may explain the long-term behavioral and cognitive disorders observed in survivors of critical illness. The hippocampus is highly vulnerable to sepsis, and structural and functional changes may underlie the development of depression, anxiety, and PTSD. ${ }^{40}$ The amygdala is also affected by microglial activation during sepsis. Sepsis injuries in the amygdala and their interaction with the prefrontal cortex, hypothalamus or brainstem may also be responsible for psychological and behavioral consequences. ${ }^{41}$

While sepsis causes neuropsychiatric symptoms, no single variable/biomarker can explain their development. Previous attempts to define a single post-sepsis neuropsychiatric syndrome biomarker have failed, reinforcing the complex and heterogeneous nature of sepsis. Given the substantial morbidity and mortality from sepsis each year, it is imperative to better define and understand its sequelae and provide adequate prevention and treatment. This understanding is essential to improving clinical and functional outcomes.

Our study has several limitations, including its sample size and the significant follow-up losses. The former can be explained by the challenge of recruiting patients according to stringent criteria. Only 5\% (33 of 658) of the screened patients met the study inclusion criteria, with many excluded due to tracheostomy, which prevented their assessment within $24 \mathrm{~h}$ of ICU discharge. The unstable post-sepsis medical conditions and deaths partially explain the significant follow-up losses. Although the instruments used to assess PTSD, anxiety and depressive symptoms are widely used in ICU studies, they cannot diagnose PTSD, anxiety, or depression. Our study was conducted at a single center and may not be generalizable all ICU sepsis survivors. Since we could not assess PTSD, depression, and anxiety prior to ICU admission, we cannot categorically state that patients had no preexisting psychiatric disorders. Finally, as in any observational study, the possibility of residual confounding remains.

In conclusion, this study provides important insight into the prevalence of and risk factors for PTSD, depression and anxiety symptoms in severe sepsis and septic shock patients after ICU discharge. More than three-quarters of the patients presented clinically relevant psychiatric symptoms during the follow-up period. Since sepsis is a major contributor to the global burden of disease and the burden of sepsis is most likely higher in low- and middleincome countries, a better understanding of the consequences of sepsis and identification of patients at risk for PTSD, depression and anxiety may allow better planning for their follow-up and treatment.

\section{Disclosure}

The authors report no conflicts of interest.

\section{References}

1 Singer M, Deutschman CS, Seymour CW, Shankar-Hari M, Annane $\mathrm{D}$, Bauer $\mathrm{M}$, et al. The third international consensus definitions for sepsis and septic shock (Sepsis-3). JAMA. 2016;315:801-10.

2 Fleischmann C, Scherag A, Adhikari NK, Hartog CS, Tsaganos T, Schlattmann P, et al. Assessment of global incidence and mortality of hospital-treated sepsis. Current estimates and limitations. Am J Respir Crit Care Med. 2016;193:259-72.

3 Iwashyna TJ, Cooke CR, Wunsch H, Kahn JM. Population burden of long-term survivorship after severe sepsis in older Americans. J Am Geriatr Soc. 2012;60:1070-7.

4 Yende S, Austin S, Rhodes A, Finfer S, Opal S, Thompson T, et al. Long-term quality of life among survivors of severe sepsis: analyses of two international trials. Crit Care Med. 2016;44:1461-7.

5 Jones C, Griffiths RD. Mental and physical disability after sepsis. Minerva Anestesiol. 2013;79:1306-12.

6 Jaenichen D, Brunkhorst FM, Strauß B, Rosendahl J. [Physical and mental long-term sequelae following intensive care of severe sepsis in patients and relatives]. Psychother Psychosom Med Psychol. 2012;62:335-43. 
7 Levy MM, Fink MP, Marshall JC, Abraham E, Angus D, Cook D, et al. $2001 \mathrm{SCCM} / \mathrm{ESICM} / \mathrm{ACCP} / \mathrm{ATS} / \mathrm{SIS}$ international sepsis definitions conference. Crit Care Med. 2003;31:1250-6.

8 Jorm AF, Korten AE. Assessment of cognitive decline in the elderly by informant interview. Br J Psychiatry. 1988;152:209-13.

9 Jorm AF. The informant questionnaire on cognitive decline in the elderly (IQCODE): a review. Int Psychogeriatr. 2004;16:275-93.

10 Weathers FW, Litz BT, Herman D, Huska J, Keane T. PTSD CheckList - Civilian Version (PCL-C) [Internet]. 1994 [cited 2020 Jul 28]. www. mirecc.va.gov/docs/visn6/3_PTSD_CheckList_and_Scoring.pdf

11 Beck AT, Steer RA, Brown GK, Beck AT, Steer RA, Brown G. Manual for the Beck depression inventory-II. San Antonio: Psychol Corp; 1996.

12 Starosta AJ, Brenner LA, Beck AT, Steer RA. Beck Anxiety Inventory Manual. Evol Ecol. 1990;15:501-20.

13 R Core Team. R: A language and environment for statistical computing. Vienna: R Foundation for Statistical Computing; 2013.

14 Streck EL, Comim CM, Barichello T, Quevedo J. The septic brain. Neurochem Res. 2008;33:2171-7.

15 Davydow DS, Hough CL, Langa KM, Iwashyna TJ. Presepsis depressive symptoms are associated with incident cognitive impairment in survivors of severe sepsis: a prospective cohort study of older Americans. J Am Geriatr Soc. 2012;60:2290-6.

16 Battle C, James K, Temblett P. Depression following critical illness: analysis of incidence and risk factors. J Intensive Care Soc. 2015;16:105-8.

17 Kessler RC, Birnbaum H, Bromet E, Hwang I, Sampson N, Shahly V. Age differences in major depression: results from the National Comorbidity Survey Replication (NCS-R). Psychol Med. 2010;40:225-37.

18 Zill P, Baghai TC, Engel R, Zwanzger P, Schüle C, Minov C, et al. Beta-1-adrenergic receptor gene in major depression: influence on antidepressant treatment response. Am J Med Genet Part B Neuropsychiatr Genet. 2003;120B:85-9.

19 Rivero G, Gabilondo AM, García-Sevilla JA, La Harpe R, Callado LF, Meana JJ. Increased $\alpha 2$ - and $\beta 1$-adrenoceptor densities in postmortem brain of subjects with depression: differential effect of antidepressant treatment. J Affect Disord. 2014;167:343-50.

20 Lasselin J, Elsenbruch S, Lekander M, Axelsson J, Karshikoff B, Grigoleit JS, et al. Mood disturbance during experimental endotoxemia: predictors of state anxiety as a psychological component of sickness behavior. Brain Behav Immun. 2016;57:30-7.

21 Budden MG. A comparative study of haloperidol and diazepam in the treatment of anxiety. Curr Med Res Opin. 1979;5:759-65.

22 Baldwin D, Woods R, Lawson R, Taylor D. Efficacy of drug treatments for generalised anxiety disorder: systematic review and metaanalysis. BMJ. 2011;342:d1199.

23 Baldwin DS, Anderson IM, Nutt DJ, Allgulander C, Bandelow B, den Boer JA, et al. Evidence-based pharmacological treatment of anxiety disorders, post-traumatic stress disorder and obsessive-compulsive disorder: a revision of the 2005 guidelines from the British Association for Psychopharmacology. J Psychopharmacol. 2014;28:403-39.

24 Wintermann GB, Rosendahl J, Weidner K, Strauß B, Petrowski K. Risk factors of delayed onset posttraumatic stress disorder in chronically critically ill patients. J Nerv Ment Dis. 2017;205:780-7.

25 Davydow DS, Gifford JM, Desai SV, Needham DM, Bienvenu OJ. Posttraumatic stress disorder in general intensive care unit survivors: a systematic review. Gen Hosp Psychiatry. 2008;30:421-34.
26 Sojka P, Stålnacke BM, Björnstig U, Karlsson K. One-year follow-up of patients with mild traumatic brain injury: Occurrence of post-traumatic stress-related symptoms at follow-up and serum levels of cortisol, S-100B and neuron-specific enolase in acute phase. Brain Inj. 2006;20:613-20.

27 Caspani G, Burcher GC, Garralda ME, Cooper M, Pierce CM, Als LC et al. Inflammation and psychopathology in children following PICU admission: an exploratory study. Evid Based Ment Heal. 2018;21: 139LP-44.

28 Sorci G, Bianchi R, Riuzzi F, Tubaro C, Arcuri C, Giambanco I, et al. S100B protein, a damage-associated molecular pattern protein in the brain and heart, and beyond. Cardiovasc Psychiatry Neurol. 2010; 2010:656481.

29 Boer KR, van Ruler O, van Emmerik AA, Sprangers MA, de Rooij SE, Vroom $\mathrm{MB}$, et al. Factors associated with posttraumatic stress symptoms in a prospective cohort of patients after abdominal sepsis: a nomogram. Intensive Care Med. 2008;34:664-74.

30 Rattray JE, Johnston M, Wildsmith JA. Predictors of emotional outcomes of intensive care. Anaesthesia. 2005;60:1085-092.

31 Clausen AN, Francisco AJ, Thelen J, Bruce J, Martin LE, McDowd J et al. PTSD and cognitive symptoms relate to inhibition-related prefrontal activation and functional connectivity. Depress Anxiety. 2017;34:427-36.

32 Zlomuzica A, Woud ML, Machulska A, Kleimt K, Dietrich L, Wolf OT, et al. Deficits in episodic memory and mental time travel in patients with post-traumatic stress disorder. Prog Neuropsychopharmacol Biol Psychiatry. 2018;83:42-54.

33 Hayes JP, VanElzakker MB, Shin LM. Emotion and cognition interactions in PTSD: a review of neurocognitive and neuroimaging studies. Front Integr Neurosci. 2012;6:89.

34 Hermelink K, Voigt V, Kaste J, Neufeld F, Wuerstlein R, Bühner M, et al. Elucidating pretreatment cognitive impairment in breast cancer patients: the impact of cancer-related post-traumatic stress. J Natl Cancer Inst. 2015;107:djv099.

35 Bauer ME, Teixeira AL. Inflammation in psychiatric disorders: what comes first? Ann N Y Acad Sci. 2019;1437:57-67.

36 Thibaut F. Neuroinflammation: new vistas for neuropsychiatric research. Dialogues Clin Neurosci. 2017;19:3-4.

37 Benros ME, Waltoft BL, Nordentoft M, Ostergaard SD, Eaton WW, Krogh J, et al. Autoimmune diseases and severe infections as risk factors for mood disorders: a nationwide study. JAMA Psychiatry. 2013;70:812-20.

38 Andersson NW, Goodwin RD, Okkels N, Gustafsson LN, Taha F, Cole SW, et al. Depression and the risk of severe infections: prospective analyses on a nationwide representative sample. Int J Epidemiol. 2016;45:131-9.

39 Mostel Z, Perl A, Marck M, Mehdi SF, Lowell B, Bathija S, et al. Postsepsis syndrome - an evolving entity that afflicts survivors of sepsis. Mol Med. 2019;26:6.

40 Sonneville R, Verdonk F, Rauturier C, Klein IF, Wolff M, Annane D, et al. Understanding brain dysfunction in sepsis. Ann Intensive Care. 2013;3:15.

41 Heming N, Mazeraud A, Verdonk F, Bozza FA, Chrétien F, Sharshar T. Neuroanatomy of sepsis-associated encephalopathy. Crit Care. 2017;21:65. 